\title{
On a heuristic point of view concerning the motion of matter. From random metric to Schrödinger equation.
}

\author{
Emilio Santos \\ Departamento de Física. Universidad de Cantabria. Santander. Spain
}

\begin{abstract}
The motion of a particle is studied in a random space-time metric, using a non-relativistic approximation. The randomness induces a diffusion of the particle in coordinate space. Hence it is shown that the evolution of the probability density of the particle's positions is given by Schrödinger equation.

PACS 03.65.Bz; 04.60.-m
\end{abstract}

\section{Introduction}

Einstein never accepted quantum mechanics as a fundamental theory of nature. Furthermore, he believed that a fundamental theory could not be found starting from the current form of quantum mechanics (e.g. by adding hidden variables) but within a completely different framework, probably that of general relativity. In his own words: "I do not believe that quantum mechanics will be the starting point in the search for this basis just as one cannot arrive at the foundations of mechanics from thermodynamics or statistical mechanics" [1. The present paper attempts to explore the possible derivation of a fundamental theory of motion in agreement with Einstein's expectations. Here I shall restrict the study to motion with small velocity in order to obtain a non-relativistic approximation. As we shall see, although the starting point is different, the theory has some similarity with the de 
Broglie-Bohm hidden-variables theory (or Bohmian mechanics) 2]. In particular, both theories assume the existence of trajectories for the particles. The difference is that Bohmian mechanics rests upon the hydrodynamic interpretation of Schrödinger equation, in which particle trajectories never cross each other. In contrast, in this paper I consider that the particle's motion consists of a random motion superimposed to a smooth one. Thus we might speak about an aerodynamic interpretation, which makes the approach similar to stochastic mechanics 3 .

General relativity starts from the assumption that space-time may be curved. The curvature can be derived from the space-time metric, once a coordinate system is defined. The specific assumption in this paper is that the metric is random. There are several reasons for this hypothesis. Firstly, noise is quite natural in our very complex universe, therefore to assume the existence of randomness is more plausible than to assume its absence. Secondly quantum theory contains a random ingredient in the form of vacuum fluctuations. At a difference with the standard assumption of nineteenth-century physics that randomness is always associated to finite (nonzero) temperature, quantum physics of the twentieth century contains "zeropoint fields", that is some randomness even at zero Kelvin. Thus I propose that the motion of bodies should be always studied in a random space-time metric. The randomness would be specified by defining the probability distribution in the set of possible metrics, but in the present paper I shall not state that distribution and use only some assumptions about it.

\section{Motion in a random metric}

I consider a particle which is placed in $\mathbf{x}_{1}$ at time $t_{1}$ and in $\mathbf{x}_{2}$ at time $t_{2}$. It moves under the action of a potential $\mathrm{U}(\mathbf{x})$ in a space-time metric

$$
d s_{\lambda}^{2}=g_{00} c^{2} d t^{2}+2 g_{0 j} c d t d x^{j}+g_{j k} d x^{j} d x^{k}, j, k=1,2,3,
$$

where $\mathrm{g}_{00}, \mathrm{~g}_{0 j}$ and $\mathrm{g}_{i j}$ are functions of $(\lambda ; \mathbf{x}, t), c$ being the velocity of light. As appropriate for the non-relativistic approximation to be introduced later, we distinguish the time, $t$, from the space coordinates, $\left\{x^{1}, x^{2}, x^{3}\right\}$, these defining the position vector, $\mathbf{x}$. The randomness is taken into account assuming that there is a probability density, $P(\lambda), \lambda \in \Lambda$, in the set $\Lambda$ of space-time metrics. (For clarity in the physical arguments to be introduced later, I use the notation $P(\lambda) d \lambda$ for the probability distribution, without any claim of 
mathematical rigour.) Some ambiguity appears due to the freedom existing in general relativity for the choice of the coordinate system, but this ambiguity should not produce any confusion in what follows. In any case I shall assume that the choice of coordinates for every metric is made so that the potential $U(\mathbf{x})$ has the same functional form in all of them.

For given $\lambda$, that is a fixed metric, the motion of the particle would correspond to the minimum of the action

$$
\begin{gathered}
A(\lambda) \equiv \int_{t_{1}}^{t_{2}}\left\{-M c d s_{\lambda}-U(\mathbf{x}) d t\right\}=\int_{t_{1}}^{t_{2}} L(\lambda ; \mathbf{x}, \dot{\mathbf{x}}, t) d t=\min , \\
L(\lambda ; \mathbf{x}, \dot{\mathbf{x}}, t) \equiv-M c \sqrt{g_{00} c^{2}+2 g_{0 j} c \dot{x}^{j}+g_{i j} \dot{x}^{i} \dot{x}^{j}}-U(\mathbf{x}),
\end{gathered}
$$

where $\dot{x}^{j}$ are the velocities (time derivatives of the position coordinates of the particle at a given time) and $U(\mathbf{x})$ is the potential. The generalization to include forces not derived from a potential is straightforward, but it will not be considered in the present paper. An alternative procedure to (2) would be to start from the differential equation of motion for the particle in a given metric (i. e. specified by a value of $\lambda$ ) but a variational principle is a more convenient starting point for my purposes. From now on I will speak about the set of Lagrange functions (3), rather than the set of metrics, but use the same label, $\Lambda$, for both sets, which should not introduce any confusion.

In this paper I will consider only motions such that $\dot{x}^{j}<<c$, and consequently I shall use a non-relativistic approximation. Thus I may replace (3) by an expansion to second order in the velocities $\dot{x}^{j}$, which gives

$L(\lambda ; \mathbf{x}, \dot{\mathbf{x}}, t)=-M\left[\sqrt{g_{00}} c^{2}+\frac{c g_{0 j}}{\sqrt{g_{00}}} \dot{x}^{j}+\left(\frac{g_{j k}}{2 \sqrt{g_{00}}}-\frac{g_{0 j} g_{0 k}}{2\left(\sqrt{g_{00}}\right)^{3}}\right) \dot{x}^{j} \dot{x}^{k}\right]-U(\mathbf{x})$.

For later convenience I define the "mean Lagrange function $L_{0}$ ", which might be obtained by an average over $\lambda$ of (4), for fixed $\mathbf{x}, \dot{\mathbf{x}}$ and $t$. It is

$$
L_{0}(\mathbf{x}, \dot{\mathbf{x}}, t) \equiv \int d \lambda P(\lambda) L(\lambda ; \mathbf{x}, \dot{\mathbf{x}}, t)=-m c^{2}+\frac{1}{2} m \dot{\mathbf{x}}^{2}-U(\mathbf{x}) .
$$

The simplicity of eq.(15) is a consequence of the invariance properties which I assume for the probability distribution of metrics, that is invariance to translations, rotations, time-translations and Galilean transformations. These invariance properties allow for the renormalized mass $m$ to be different from the bare mass $M$. The Lagrange function (4) may be written as a sum

$$
L(\lambda ; \mathbf{x}, \dot{\mathbf{x}}, t)=L_{0}(\mathbf{x}, \dot{\mathbf{x}}, t)+L_{1}(\lambda ; \mathbf{x}, \dot{\mathbf{x}}, t),
$$


such that all randomness goes in the second term.

The probability distribution in the set of Lagrange functions induces a probability distribution in the set of possible paths of the particle, which may be formalized as follows. We consider the set, $N$, of paths going from $\left(\mathbf{x}_{1}, t_{1}\right)$ to $\left(\mathbf{x}_{2}, t_{2}\right)$, each path specified by the equation of motion $x^{j}(\nu ; t)$, $\nu \in N, t \in\left[t_{1}, t_{2}\right]$, where we assume that $\mathrm{x}^{j}$ as a function of $t$, for fixed $\nu$, possesses continuous second derivatives. Our problem is to find the probability distribution in $N$ corrresponding to that in $\Lambda$. Now the condition (2) defines a function, $\nu=g(\lambda)$, associating a path to every Lagrange function, which may be got from the variational problem

$$
I \equiv \int_{\Lambda} P(\lambda) \delta(\nu-g(\lambda)) d g(\lambda) \int_{t_{1}}^{t_{2}} L(\lambda ; \mathbf{x}(\nu, t), \dot{\mathbf{x}}(\nu, t), t) d t=\min ,
$$

where $P(\lambda) \geq 0$ (see (5D)). In fact, any function $g(\lambda)$ which does not associate to every $\lambda$ the path giving the minimum value to the action $A(\lambda)$ (22) will lead to a value of $I$ (7) which is greater (strictly, not smaller) than the one given by the function leading to the minimum value of $I$.

In order to devise a practical method to solve the variational problem (77) I suppose that the time integral may be approximated by a sum over a finite number of times, that is $\varepsilon, 2 \varepsilon, 3 \varepsilon, \ldots$ with $\varepsilon$ small enough. For this we require the position, $\mathbf{y}$, and the velocity, $\mathbf{w}$, corresponding to every path at every one of the discrete times. Thus we write, instead of (7), the variational condition

$$
\begin{aligned}
J & \equiv \int_{\Lambda} P(\lambda) \delta(\nu-g(\lambda)) d g(\lambda) K=\min \\
K & \equiv \int_{t_{1}}^{t_{2}} d t \int d^{3} \mathbf{y} \int d^{3} \mathbf{w} \delta(\mathbf{y}-\mathbf{x}(\nu, t)) \delta(\mathbf{w}-\dot{\mathbf{x}}(\nu, t)) L(\lambda ; \mathbf{y}, \mathbf{w}, t),
\end{aligned}
$$

where $\delta()$ is the three-dimensional Dirac's delta and the unknown function to be found is $g(\lambda)$. Now for any $g(\lambda)$ (not necessarily the one providing the minimum value of $J$ ) we may define a probability distribution, $f_{g}(\mathbf{y}, \mathbf{w}, t)$, in the phase space of positions and velocities via the integral

$$
f_{g}(\mathbf{y}, \mathbf{w}, t)=\int_{\Lambda} P(\lambda) \delta(\nu-g(\lambda)) d g(\lambda) \delta(\mathbf{y}-\mathbf{x}(\nu, t)) \delta(\mathbf{w}-\dot{\mathbf{x}}(\nu, t)) .
$$

Now our problem is to get, from the variational condition (8), another one involving the phase-space distribution $f_{g}(\mathbf{y}, \mathbf{w}, t)$, rather than the function 
$g$, if this is possible. The use of the phase-space distribution leads to a fluiddynamical picture of the motion where the actual particle is replaced by a statistical ensemble of particles whose phase-space density is $f(\mathbf{y}, \mathbf{w}, t)$.

A case where the variational problem (86) may be easily written in terms of a phase-space distribution corresponds to $L$ not depending on $\lambda$, that is when the Lagrange function is not random. I shall solve this rather trivial case in the hope that it may provide a clue for a more general method to be developed in the next section. In this case inserting (9) in (8) leads to the variational condition

$$
\int_{t_{1}}^{t_{2}} d t \int d^{3} \mathbf{x} \int d^{3} \mathbf{w} f(\mathbf{x}, \mathbf{w}, t) L(\mathbf{x}, \mathbf{w}, t)=\min .
$$

Putting the Lagrange function (15) in this variational problem gives

$$
\int_{t_{1}}^{t_{2}} d t \int d^{3} \mathbf{x} \int d^{3} \mathbf{w} f(\mathbf{x}, \mathbf{w}, t)\left[\frac{1}{2} m \mathbf{w}^{2}-U(\mathbf{x})\right]=\min
$$

where we ignore the first term of (5) which, being a constant, is irrelevant in the Lagrange function. Without any loss of generality we may write

$$
f(\mathbf{x}, \mathbf{w}, t)=\rho(\mathbf{x}, t) h(\mathbf{x}, \mathbf{w}, t),
$$

with the conditions

$$
\int h(\mathbf{x}, \mathbf{w}, t) d^{3} \mathbf{w}=1, \int \mathbf{w} h(\mathbf{x}, \mathbf{w}, t) d^{3} \mathbf{w}=\mathbf{v}(\mathbf{x}, t),
$$

so that $\rho(\mathbf{x}, t)$ is the density of particles at $(\mathbf{x}, t)$, and $\mathbf{v}(\mathbf{x}, t)$ is the mean velocity of those particles present at that space-ime point. Now I shall make the minimization of (11) in two steps. In the first one I will search for the function $h(\mathbf{x}, \mathbf{w}, t)$ making the action (111) a minimum with given $\rho(\mathbf{x}, t)$ and $\mathbf{v}(\mathbf{x}, t)$. In the second step I will find the equations of motion of these two functions.

The minimum of (11) with fixed $\rho$ and $\mathbf{v}$ requires, for every $(\mathbf{x}, t)$,

$$
\int \mathbf{w}^{2} h(\mathbf{x}, \mathbf{w}, t) d^{3} \mathbf{w}=\min
$$

with the constraints (12), which leads to

$$
h(\mathbf{x}, \mathbf{w}, t)=\delta(\mathbf{w}-\mathbf{v}(\mathbf{x}, t)),
$$


$\delta()$ being the 3-dimensional Dirac's delta. This means that all particles present at $(\mathbf{x}, t)$ possess the same velocity, that is the phase-space probability becomes

$$
f(\mathbf{x}, \mathbf{w}, t)=\rho(\mathbf{x}, t) \delta(\mathbf{w}-\mathbf{v}(\mathbf{x}, t)),
$$

which is a phase-space dependence typical of hydrodynamics. Thus density and velocity are related by the continuity equation

$$
\frac{\partial \rho}{\partial t}+\nabla \mathbf{j}=0, \mathbf{j}=\rho \mathbf{v}
$$

j being the current density. As a conclusion of the first step, the form (14) allows to state the variational condition in the form

$$
\int_{t_{1}}^{t_{2}} d t \int d^{3} \mathbf{x} \rho(\mathbf{x}, t)\left[\frac{1}{2} m \mathbf{v}(\mathbf{x}, t)^{2}-U(\mathbf{x})\right]=\min
$$

with the constraint (15) .

Now we derive the equations of motion of $\rho$ and $\mathbf{v}$ from (16), which is straightforward. We introduce the condition (15) in the variational problem by means of the Lagrange multiplier $S(\mathbf{x}, t)$ and get

$$
\delta \int_{t_{1}}^{t_{2}} d t \int d^{3} \mathbf{x}\left\{\rho\left[\frac{1}{2} m \mathbf{v}^{2}-U(\mathbf{x})\right]+S\left[\frac{\partial \rho}{\partial t}+\nabla(\rho \mathbf{v})\right]\right\}=0
$$

where the variation of the action must be zero for any independent variations of $\rho, \mathbf{v}$ and $S$ ( the variation of $S$ just reproduces (15)). The function $S$ must fulfil the condition

$$
S\left(\mathbf{x}_{1}, t_{1}\right)=S\left(\mathbf{x}_{2}, t_{2}\right)
$$

relating the initial and the final times and positions. From the variation of $\mathbf{v}$ in (17) we obtain, after an integration by parts,

$$
\mathbf{v}=\frac{1}{m} \nabla S
$$

(The integrated term is zero because $\rho$ vanishes at infinity). If (19) is inserted in (17) we get, after two appropriate integrations by parts,

$$
\delta \int_{t_{1}}^{t_{2}} d t \int d^{3} \mathbf{x} \rho\left[\frac{1}{2 m}(\nabla S)^{2}+U(\mathbf{x})+\frac{\partial S}{\partial t}\right]=0 .
$$


The time integration by parts requires that the integral

$$
\int S(\mathbf{x}, t) \rho(\mathbf{x}, t) d^{3} \mathbf{x}
$$

has the same value at times $t_{1}$ and $t_{2}$, which holds true in view of (18). In the variational problem (20) the variation of $\rho$ leads to the Hamilton-Jacobi equation whilst the variation of $S$ gives again the continuity eq.(15), taking (19) into account, that is

$$
\frac{\partial \rho}{\partial t}+\frac{1}{m} \nabla(\rho \nabla S)=0
$$

These results reproduce standard equations of motion in analytical mechanics as it should.

\section{Derivation of Schrödinger equation}

When $L_{1} \neq 0$, that is the metric is random, it is not obvious that the problem of the motion may be solved in terms of the two functions $\rho(\mathbf{x}, t)$ and $\mathbf{v}(\mathbf{x}, t)$. Indeed, the condition (14) will not be fulfilled in general. Nevertheless, I shall assume that such a solution of the problem is still possible provided that we use a functional of $\rho(\mathbf{x}, t)$ and $\mathbf{v}(\mathbf{x}, t)$, rather than a function as in (16) . In any case the randomness of the metric will produce randomness in the velocities of the particles arriving in position $\mathbf{x}$ at time $t$. We shall take into account that randomness modifying both the continuity eq.(15) and the variational condition (16). If $\mathbf{w}$ is the velocity of a particle placed in $\mathbf{x}$ at time $t$, the position of the particle at time $t+\Delta t$ will be, neglecting terms of order $\triangle t^{2}$ and higher,

$$
\mathbf{y}=\mathbf{x}+\mathbf{w} \Delta t+\triangle \mathbf{x}
$$

$\triangle \mathbf{x}$ being a random displacement. I propose to treat the displacement $\triangle \mathbf{x}$ as deriving from a white noise independent of time, position, and initial velocity, thus leading to the following probability distribution for the (vector) displacement $\triangle \mathbf{x}$

$$
Q(\triangle \mathbf{x})=(4 \pi D \triangle t)^{-3 / 2} \exp \left(-\frac{|\triangle \mathbf{x}|^{2}}{4 D \triangle t}\right)
$$


This assumption rests upon the hypothesis that the change of position induced by the space-time dependence of the coefficients, $g_{\mu \nu}$, of the metric is more rapid than the change induced by the external forces (deriving from the potential $U(\mathbf{x})$ ), which is plausible for a non-relativistic theory as ours. Actually, if (23) is correct, there is a finite (small) probability that the velocity of light is surpassed, but this fact is not a real problem within the non-relativistic approximation.

From (23) it is straightforward to get, from the density, $\rho(\mathbf{x}, t)$, of particles at time $t$ the density at time $t+\Delta t$. We get

$$
\rho(\mathbf{y}, t+\triangle t)=\int d^{3} \mathbf{x} \int d^{3} \mathbf{w} f(\mathbf{x}, \mathbf{w}, t) Q(\mathbf{y}-\mathbf{x}-\mathbf{w} \triangle t)
$$

where $f(\mathbf{x}, \mathbf{w}, t)$ is the phase-space probability distribution at time $t$. To first order in $\triangle t$ the integration is straightforward if we use the expansion

$$
f(\mathbf{x}, \mathbf{w}, t) \simeq f(\mathbf{y}, \mathbf{w}, t)+\sum_{j}\left(x_{j}-y_{j}\right) \frac{\partial f}{\partial y_{j}}+\frac{1}{2} \sum_{j} \sum_{k}\left(x_{j}-y_{j}\right)\left(x_{k}-y_{k}\right) \frac{\partial^{2} f}{\partial y_{j} \partial y_{k}}
$$

Inserting (25) in (24) and performing the integrals in $\mathbf{x}$ and $\mathbf{w}$ we get, after some algebra,

$$
\frac{\partial \rho}{\partial t}+\nabla \mathbf{j}=0, \mathbf{j}=\rho \mathbf{v}-D \nabla \rho
$$

where we have taken into account

$$
\int f(\mathbf{y}, \mathbf{w}, t) \mathbf{d}^{\mathbf{3}} \mathbf{w}=\rho(\mathbf{x}, \mathbf{t}), \int \mathbf{w} f(\mathbf{y}, \mathbf{w}, t) d^{3} \mathbf{w}=\rho(\mathbf{x}, t) \mathbf{v}(\mathbf{y}, t),
$$

(see (12).) Thus we arrive at the following

Proposition 1 The density, $\rho(\mathbf{x}, t)$, and the mean velocity, $\mathbf{v}(\mathbf{x}, t)$, of the particles in the statistical ensemble representing the actual particle, fulfil de continuity eq.(26).

We see that now there is a "diffusion current" (last term) in addition to the "hydrodynamical current" (compare with (15)). In order to get the substitute for (16) we introduce the random velocity $\mathbf{u}=\mathbf{w}-\mathbf{v}(\mathbf{x}, t)$, so that

$$
\left\langle\mathbf{w}^{2}\right\rangle_{\mathbf{x}, t}=\mathbf{v}(\mathbf{x}, t)^{2}+\left\langle\mathbf{u}^{2}\right\rangle_{\mathbf{x}, t}+2 \mathbf{v}(\mathbf{x}, t) \cdot\langle\mathbf{u}\rangle_{\mathbf{x}, t}
$$


where \langle\rangle$_{\mathbf{x}, t}$ means average over those particles present in $\mathbf{x}$ at time $t$. In this equation the random-velocity average, $\langle\mathbf{u}\rangle_{\mathbf{x}, t}$, is the diffusion velocity which, from (26), should be

$$
\langle\mathbf{u}\rangle_{\mathbf{x}, t}=-D \rho^{-1} \nabla \rho .
$$

The difficult problem is to calculate the random square mean velocity, $\left\langle\mathbf{u}^{2}\right\rangle_{\mathbf{x}, t}$, which is not possible without a detailed knowledge of the probability distribution of metrics. Thus I will make the most simple assumption, namely that it is a constant independent of $\mathbf{x}$ and $t$. The lack of a clear foundation for this hypothesis is certainly a weak point of the present derivation, which will be studied more carefully elsewhere. In any case, once the assumption is accepted it is straightforward to arrive at the following

Proposition 2 The mean kinetic energy of the particles (of the statistical ensemble) present at $(\mathbf{x}, t)$ is given by

$$
T(\mathbf{x}, t)=\frac{1}{2} m\left(\mathbf{v}^{2}-2 D \rho^{-1} \mathbf{v} \cdot \nabla \rho\right)+T_{0},
$$

where $T_{0}$ is a constant.

The assumption made in (23), that the change of position induced by the space-time dependence of the metric coefficients is more rapid than the change induced by the external potential, implies that the constant $T_{0}$ is rather large so that the kinetic energy is always positive. As a conclusion the action (16) should be replaced by

$$
\int_{t_{1}}^{t_{2}} d t \int d^{3} \mathbf{x} L=\min , L \equiv \frac{1}{2} m\left(\rho \mathbf{v}^{2}-2 D \mathbf{v} \cdot \nabla \rho\right)-\rho U(\mathbf{x})
$$

where we have ignored the constant $T_{0}$ which is irrelevant in the variational problem. Now we follow the same steps leading from (15) and (16) to (20). We introduce the constraint (26) in the variational problem (28) using a Lagrange parameter $\mathrm{S}$, which leads to

$$
\delta \int_{t_{1}}^{t_{2}} d t \int d^{3} \mathbf{x}\left\{L+S\left[\frac{\partial \rho}{\partial t}+\nabla(\rho \mathbf{v}-D \nabla \rho)\right]\right\}=0 .
$$

Hence the variation of $\mathbf{v}$ gives, after an integration by parts, the following relation

$$
\mathbf{v}=D \frac{\nabla \rho}{\rho}+\frac{\nabla S}{m} .
$$


When this is inserted in (26) we get the continuity equation in the form (21). On the other hand when (30) is inserted in (29) we obtain

$$
\delta \int_{t_{1}}^{t_{2}} d t \int d^{3} \mathbf{x}\left\{S \frac{\partial \rho}{\partial t}-\rho\left[\frac{1}{2 m}(\nabla S)^{2}+\frac{1}{2} m D^{2}\left(\frac{\nabla \rho}{\rho}\right)^{2}+U(\mathbf{x})\right]\right\}=0
$$

where we have ignored two terms whose sum equals the divergence of the vector field $D S \nabla \rho$. We must assume that this vector field vanishes at infinity whence the integral of its divergence is zero.

It is remarkable that, although we have started from two expressions, (26) and (28), neither of which is invariant under the reversal of time, the Lagrange density in (31) is time-reversal invariant in the sense that the operation $(t \rightarrow-t)$ is equivalent to just changing the sign of the auxiliary function $S$, which does not change the physics. In (31) it is easy to see that the variation of $\rho$ leads to

$$
\frac{1}{2 m}(\nabla S)^{2}+U(\mathbf{x})+\frac{\partial S}{\partial t}-2 m D^{2} \frac{\nabla^{2} \sqrt{\rho}}{\sqrt{\rho}}=0,
$$

where we have assumed that the space-time integral of $\partial(S \rho) / \partial t$ vanishes, a hypothesis already made in the previous section (see below eq.(20) ). As is well known the continuity eq.(21) and the dynamical eq.(32) may be obtained by separating the real and imaginary parts in the Schrödinger equation

$$
i \hbar \frac{\partial \Psi}{\partial t}=-\frac{\hbar^{2}}{2 m} \nabla^{2} \Psi+U \Psi
$$

provided that we identify

$$
\hbar \equiv 2 m D, \Psi \equiv \sqrt{\rho} \exp \left(\frac{i S}{\hbar}\right)
$$

\section{Discussion}

The derivation of Schrödinger equation given in this paper might be considered a step in the direction of Einstein's expectations, as commented in the introduction. Alternatively it may be seen as just a new derivation from formal assumptions (the two propositions of the previous section) devoid of any deep physical meaning. I cannot argue too strongly in favour of the former 
possibility, and this is why I include the word "heuristic" in the title of the paper.

In our derivation the "wave-function" $\Psi$ is just a mathematical function, with range in the complex numbers, whose modulus gives information about the probability density of the particle position and whose phase is related to the particle velocity via eq.(30). In comparison with the standard Hamilton-Jacobi equation, see (201), eq.(32) contains a term deriving from the fact that the space-time metric differs from the standard one of Minkowski space. Thus the metric plays the role of the "guiding wave" in the de Broglie-Bohm theory 2]. It is not contrary to the intuition that this "wave" may modify the scattering cross sections, making them different from those derived from classical mechanics. Also it is easy to understand the existence of stationary states of electrons in atoms, as a balance between the attraction by the nucleus and the diffusion caused by the random metric. The picture is here similar to that provided by stochastic electrodynamics 4 with the gravitational field (the non-Minkowskian metric) substituted for the electromagnetic radiation. More difficult is to understand intuitively how the non-Minkowskian metric may give rise to the observed sharp spectral lines of atoms or to the interference fringes in two-slit experiments. If the latter two effects derive from the metric, the mechanism is not clear from the derivation here presented.

A question which arises is whether the assumption of a random metric is really necessary for the two hypotheses introduced in the previous section. Indeed, derivations of Schrödinger equation from the hypothesis of a random motion, not involving the space-time metric, have been recurrently proposed during the last 50 years or more 3, 4]. In my view there are three reasons why the randomness of the metric is relevant in the derivation given in the present paper. The first one is the fundamental character of the space-time background, as emphasized in the introduction. The second reason is the existence of a diffusion in coordinate space rather than in velocity space, as would be more natural in any approach starting from Newtonian mechanics. Also, if the origin of the random motion is gravitational, dissipative effects may be negligible. The third reason is that metric randomness makes plausible a formulation starting from a variational condition, due to the fundamental role which geodesics play in general relativity. Nevertheless the fact that Planck's $\hbar$ is a universal constant barely follows from the existence of random metrics. Indeed within general relativity (or Newtonian gravity) the motions (of small particles) are independent of the mass, which suggests 
that the diffusion constant $D$ (see (34)), rather than $\hbar$, should be universal. I have no clear response to this objection. I may mention only the fact that, when we consider the diffusion of a system consisting of $n$ particles of equal mass, $m$, it may be shown that the diffusion constant of the center of mass of the system is $D / n$ if the particles diffuse independently. In this case the product, $m D$, of the mass times the diffusion parameter is the same for the individual particles and for the center of mass of the system, independently of $n$.

An interesting question is whether the derived equations ((21) and (32)) are equivalent to Schrödinger equation. The answer is in the negative. The reason is that the derivation of the previous section implies that the function $S(\mathbf{x}, t)$ must be single-valued whilst in Schrödinger theory the wavefunction $\Psi(\mathbf{x}, t)$ is single-valued, which just requires that $S(\mathbf{x}, t)$ changes by an integer multiple of $2 \pi \hbar$ along any closed line. Actually both assumptions are equivalent, by continuity, if the region of definition of $S$ (or $\Psi$ ) is simply connected. However it is currently assumed that there are experimental situations where this is not the case. In particular the popular two-slit experiments, which have already been performed with electrons, neutrons, atoms and even molecules, are currently analyzed assuming the existence of a region forbidden to the particles, namely the one occupied by the screen with the slits, so that the region allowed to the particles is not simply connected. However it might be possible to study these experiments replacing the screen by a high, but finite, repulsive potential so that the region is simply connected. Another case where the region of interest is not simply connected corresponds to some excited states of atoms. In this case the wavefunction may become singular at the origin (the position of the nucleus). For instance the wavefunction of the states with quantum numbers $l=m \neq 0$ contains the factor $\exp (i l \phi)$ and the function $S$ changes by $2 \pi l \hbar$ in a rotation by $2 \pi$. A related problem is the existence of nodal surfaces in some solutions of Schrödinger equation, that is surfaces where $\rho=0$ but $\mathbf{v} \neq 0$. These solutions are unphysical in our approach. In summary every physical solution of (21) and (32) is a solution of Schrödinger equation (33), but there are solutions of the latter which are not solutions of the former. This seems to imply that the formalism here developed cannot agree with the empirical evidence. Nevertheless I question the current wisdom that all solutions of the Schrödinger equation are really necessary for the interpretation of the experiments, but this point will not be analyzed further here.

In any case the formulation here presented allows for an interpretation of 
Schrödinger equation in terms of trajectories, which may be useful in some applications. In this sense the formulation is similar in spirit to Bohmian mechanics 2. However our trajectories present a random element and so the picture achieved is actually more similar to stochastic mechanics. But stochastic mechanics is currently understood as fully equivalent to Schrödinger theory, which leads to counterintuitive behaviour like the existence of nodal surfaces mentioned above. For this reason it is usually considered as just a formal approach to quantum mechanics rather than a different physical theory 3 .

The formalism here presented might be extended to many-particle systems by replacing the three dimensional space by the $3 N$ dimensional configuration space of $N$ particles. However all particles will move in the same space-time metric, which would induce correlations in the motion, in addition to those derived from the possible inter-particle forces. Thus the generalization is not trivial. Incidentally, I guess that the said correlations might be related to Bose statistics, but this point will not be discussed further here.

In summary, the formalism here developed allows an interpretation of Schródinger equation in terms of particle trajectories, which may have some interest. However there are great difficulties to take it as a physical theory underlying quantum mechanics.

\section{References}

[1] A. Pais, Rev. Mod. Phys. 51, 863 (1979).

[2] P. R. Holland, The Quantum Theory of Motion. Cambridge University Press. Cambridge, 2004.

[3] E. Nelson, Quantum Fluctuations, Princeton University Press. Princeton, 1985 .

[4] L de la Peña and A. M. Cetto, The quantum dice. An introduction to stochastic electrodynamics. Kluwer Academic. Dordrecht, 1996. 